NASA Technical Memorandum 106813

ICOMP-94-31; AIAA-94-3369

\title{
A Three-Dimensional Structured/Unstructured Hybrid Navier-Stokes Method for Turbine Blade Rows
}

F.-L. Tsung and J. Loellbach

Institute for Computational Mechanics in Propulsion

National Aeronautics and Space Administration

Lewis Research Center

Cleveland, Ohio

O. Kwon

NYMA, Inc.

Engineering Services Division

Brook Park, Ohio

C. Hah

National Aeronautics and Space Administration

Lewis Research Center

Cleveland, Ohio

Prepared for the

30th Joint Propulsion Conference and Exhibit cosponsored by AIAA, ASME, SAE, and ASEE Indianapolis, Indiana, June 27-29, 1994

National Aeronautics and

Space Administration

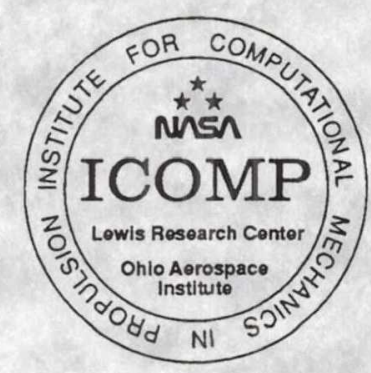




\title{
A THREE-DIMENSIONAL STRUCTURED/UNSTRUCTURED HYBRID NAVIER-STOKES METHOD FOR TURBINE BLADE ROWS
}

\author{
F.-L. Tsung* and J. Loellbach* \\ Institute for Computational Mechanics in Propulsion $\S$ \\ NASA Lewis Research Center \\ Cleveland, Ohio \\ O. Kwon ${ }^{\dagger}$ \\ NYMA, Inc. NASA Lewis Research Center Group \\ Brook Park, Ohio \\ C. $\mathrm{Hah}^{\ddagger}$ \\ NASA Lewis Research Center \\ Cleveland, Ohio
}

\begin{abstract}
A three-dimensional viscous structured / unstructured hybrid scheme has been developed for numerical computation of high Reynolds number turbomachinery flows. The procedure allows an efficient structured solver to be employed in the densely clustered, high aspect-ratio grid around the viscous regions near solid surfaces, while employing an unstructured solver elsewhere in the flow domain to add flexibility in mesh generation. Test results for an inviscid flow over an external transonic wing and a Navier-Stokes flow for an internal annular cascade are presented.
\end{abstract}

\section{Introduction}

In modern turbomachinery designs, the rotor and stator blade rows often possess extreme turning angles such that the flow direction deviates greatly from the axial direction. These geometries frequently cause difficulties in generating structured meshes [1]. In order to impose point-to-point periodicity on periodic boundaries in the domain, the computational

* Senior Research Associate, Member AIAA

$\dagger$ Research Engineer, Turbomachinery Analysis

Section, Member AIAA

¥ Senior Scientist, Internal Fluid Mechanics Division, Member AIAA

$\S$ located at the Ohio Aerospace Institute adjacent to the NASA Lewis Research Center in Cleveland, Ohio

This paper is declared a work of the U.S. Government and is not subject to copyright protection in the United States. grids are typically forced into highly skewed shapes that decrease the accuracy of the solvers. One remedy for reducing the grid skewness is to forego the pointto-point correspondence on the periodic boundaries and to interpolate solution values where necessary. The disadvantage of the non-periodic grid approach is that the local density of grid lines on either side of a periodic boundary may be vastly different. Hence, flow features resolved on one side of the domain boundary may be diffused or lost when interpolated to a coarser grid on the other side. Another solution to grid skewness is to use unstructured meshes. However, the cost and technology of present day unstructured solvers still leave much room for improvement.

The computation of both inviscid and viscous flows on unstructured triangular meshes in two dimensions, and tetrahedral meshes in three dimensions, has matured significantly in recent years [2-7]. The ability of unstructured solvers to handle complex geometries has proven to be valuable for many computations. However, the geometric flexibility of unstructured grid solvers is also the source of their disadvantages when compared to structured solvers. Due to the naturally-ordered data connectivity of structured grids, structured solvers require much less memory (especially for implicit schemes), implicit structured solvers are straight forward to code, and turbulence modeling is easier to implement.

The shortcomings of both structured and unstructured methodologies are continuously being addressed and improved. In the meantime, however, it is possible to take advantage of the best properties of both methodologies. In the present paper, a solution procedure which couples an efficient 
structured solver with an unstructured solver is presented. The hybrid procedure takes advantage of the computational efficiency of structured codes and, at the same time, is able to benefit from the geometric flexibility of unstructured solvers. With the hybrid approach, densely packed structured grids can be placed in the highly viscous regions near solid surfaces, and unstructured grids can be used away from solid surfaces [8-10]. This approach can avoid the severe grid skewness commonly experienced by fully structured grids around turbine blades with high turning angles. The procedure has various other applications as well, such as providing a means of connecting multi-body geometries for Chimera-type grids to insure node-to-node correspondence [11-12].

The strategy of coupling structured and unstructured methods has been implemented for twodimensional turbomachinery computations by many researchers in the past [13-15]. The present work extends this strategy to three-dimensional turbomachinery flows. The hybrid solution technique is first tested for an external fixed-wing transonic flow case and the result is compared with a structured solution for validation. The code is then applied to an annular cascade and the result is compared with experimental data.

\section{Formulation}

In this section, the solution procedure for the unstructured solver is first described, followed by the procedure for the structured solver. Since both the structured and the unstructured solvers used in this paper have been previously documented, only brief outlines of the mathematical and numerical formulations are given. Lastly, the coupling procedure used to integrate the two solvers is detailed.

\section{Unstructured Solver}

\section{Governing Equation}

The time dependent, Reynolds averaged, compressible Navier-Stokes equations, which express the conservation of mass, momentum, and energy, are solved. The turbulence viscosity is calculated using the high Reynolds number turbulence model of Launder and Spalding [16]. The equations of motion, written in an integral form for a bounded domain $\Omega$ with a boundary $\partial \Omega$, are

$$
\begin{aligned}
& \frac{\partial}{\partial t} \iiint_{\Omega} \mathbf{Q} d V+\iint_{\partial \Omega} \mathbf{F}(\mathbf{Q}) \cdot \hat{n} d S \\
= & \iint_{\partial \Omega} \mathbf{G}(\mathbf{Q}) \cdot \hat{n} d S+\iiint_{\Omega} \mathbf{S}(\mathbf{Q}) d V
\end{aligned}
$$

where $\mathbf{Q}$ is the unknown vector containing the conserved properties

$$
\mathbf{Q}=\left\{\rho, \rho u, \rho v, \rho w, e_{0}, \rho k, \rho \varepsilon\right\}^{\mathrm{T}}
$$

In the above equation, $\rho$ is the fluid density. $u, v$, and $w$ are the Cartesian velocity components in $x, y$, and $z$ directions, respectively. $e_{o}$ is the total energy per unit volume. The turbulent kinetic energy and turbulent kinetic energy dissipation rate are represented by $k$ and $\varepsilon$. The vector $\mathbf{F}$ is the convective flux term, $\mathbf{G}$ is the viscous term, and $\mathbf{S}$ is the source term containing the production and destruction of turbulent kinetic energy. A complete description of the governing equations is presented in Reference [17] and will not be detailed here for brevity.

\section{Inviscid Flux Spatial Discretization}

The inviscid flux across each cell face $\kappa$ is computed using Roe's flux-difference splitting formula [18]

$$
\mathbf{F}_{\boldsymbol{K}}=\frac{1}{2}\left[\mathbf{F}\left(\mathbf{Q}_{L}\right)+\mathbf{F}\left(\mathbf{Q}_{R}\right)-|\tilde{\mathbf{A}}|\left(\mathbf{Q}_{R}-\mathbf{Q}_{L}\right)\right]_{\boldsymbol{K}}
$$

Here, $\mathbf{Q}_{L}$ and $\mathbf{Q}_{R}$ are the state variables to the left and right of the interface $\kappa$. The matrix $\tilde{\mathbf{A}}$ is computed by evaluating

$$
\mathbf{A} \equiv \frac{\partial \mathbf{F}}{\partial \mathbf{Q}}
$$

with Roe-averaged quantities so that

$$
\mathbf{F}\left(\mathbf{Q}_{R}\right)-\mathbf{F}\left(\mathbf{Q}_{L}\right)=\tilde{\mathbf{A}}\left[\mathbf{Q}_{R}-\mathbf{Q}_{L}\right]
$$

is satisfied.

For a first-order scheme, the primitive variables at each cell face are set equal to the cell-centered averages on either side of the face. For a higher-order scheme, estimation of the state at each face is achieved by interpolating the solution at each time step with a Taylor series expansion in the neighborhood of each cell center. The cell-averaged 
solution gradients required at the cell center for the expansions are computed using Gauss' theorem by evaluating the surface integral for the closed surface of the tetrahedra. This process can be simplified using geometric invariant features of tetrahedra [4]. The resulting second-order formula for the flow state at each cell face can be written as

$$
q_{f_{1,2,3}}=q_{i}+\frac{1}{4}\left[\frac{1}{3}\left(q_{n_{1}}+q_{n_{2}}+q_{n_{3}}\right)-q_{n_{4}}\right]
$$

where the subscripts $n_{1}, n_{2}, n_{3}$ denote the nodes comprising face $f_{1,2,3}$ of cell $i$, and $n_{4}$ corresponds to the opposite node. The expansion also requires the nodal value of the solution, which can be computed from the surrounding cell center data using a secondorder accurate pseudo-Laplacian averaging procedure as suggested by Homes and Connell [19]. The threedimensional extension by Frink [20] is adopted in the present calculations.

The convective terms of the turbulence equations are calculated using a first-order accurate scheme in the present paper to reduce the computational cost and to ensure the numerical stability of the time integration [21].

\section{Viscous Flux Spatial Discretization}

The evaluation of the viscous term $\mathbf{G}$ requires first derivatives of the velocity, temperature, and $k-\varepsilon$ values at the cell faces. They are achieved by first evaluating the gradient of each required flow quantity at the cell center from the known primitive variables at each time step. The gradient of the desired quantity is obtained by applying the gradient theorem,

$$
\nabla \phi_{n}=\frac{1}{V_{\Omega}} \oint_{\partial \Omega} \phi \hat{n} d S
$$

where $\Omega$ represents the volume of the domain over which the theorem is applied. The scalar quantity $\phi$ can be the three components of velocity, the temperature, or turbulence quantities. In the present calculations, the integral domain is defined as the individual tetrahedral cell, and the surrounding surface area $\partial \Omega$ consists of the four triangular surfaces covering the cell. This formulation is consistent with the numerical procedure of evaluating the convective fluxes of the present cell-centered scheme.
Once the gradients of the desired quantities are known at the cell center, nodal values are calculated using the pseudo-Laplacian averaging mentioned earlier for the convective terms. The flux through each of the triangular faces in equation (7) is obtained by averaging the three nodal values for the triangle. Once the gradients of the primitive variables are obtained, the shear stresses and can be calculated, from which $\mathbf{G}$ is evaluated at the cell center. The nodal values of these quantities are calculated once again by applying the pseudo-Laplacian averaging of the surrounding cell center values. The surface flux of these quantities in equation (1) is obtained by taking the average of the three nodal values for each triangular face of each cell.

\section{Time Integration}

The seven equations of motion are integrated in time using an explicit 3-stage Runge-Kutta scheme developed by Jameson et al. [22]. The inviscid fluxes are evaluated at each time stage using values of transport variables obtained at the previous stage of the scheme rather then using values from the previous iteration [23]. The viscous dissipation and the source terms are evaluated prior to the first stage and remain constant within that time iteration.

Local time stepping and implicit residual smoothing are used to accelerate the convergence to steady state. Inclusion of the viscous terms in the residual smoothing procedure was essential to obtain a stable and convergent solution. The implicit smoothing is also applied to the turbulence equations.

\section{Structured Solver}

The structured Navier-Stokes analysis in the present procedure uses a center-differenced, finitedifference scheme. The code was originally a singleblock external flow solver developed for analysis of fixed and rotary wings [24]. Multi-block capability has since been added to the code and additional boundary condition routines have also been implemented for internal flow analysis.

The structured code solves the unsteady, compressible, Reynolds-averaged Navier-Stokes equation in strong conservation form for curvilinear coordinates

$$
\frac{\partial \mathbf{Q}}{\partial \tau}+\frac{\partial(\mathbf{E}-\mathbf{E v})}{\partial \xi}+\frac{\partial(\mathbf{F}-\mathbf{F v})}{\partial \eta}+\frac{\partial(\mathbf{G}-\mathbf{G} \mathbf{v})}{\partial \zeta}=0
$$


Here, $\mathbf{Q}$ is the vector of conserved variables divided by the Jacobian of the coordinate transformation. Five unknowns are solved instead of seven as in the unstructured solver since Baldwin-Lomax algebraic [25] turbulence model is used for the structured solver.

\section{Time Integration}

The solution vector is integrated in time using the implicit Euler method, which is first-order accurate in time. The nonlinear inviscid flux vectors are linearized at every time level about their values at the previous time level using Taylor expansions, e.g.

$$
\begin{aligned}
\mathbf{E}^{n+1} & =\mathbf{E}^{n}+\frac{\partial \mathbf{E}^{n}}{\partial \mathbf{Q}} \Delta \mathbf{Q}^{n+1}+\mathrm{O}\left(\Delta \tau^{2}\right) \\
& \approx \mathbf{E}^{n}+\mathbf{A}^{n} \Delta \mathbf{Q}^{n+1}
\end{aligned}
$$

The viscous terms are evaluated explicitly. Explicit treatment of the viscous terms still permits the use of large time steps since the Reynolds numbers of interest here are fairly large. To further reduce computational time and memory, the radial/spanwise flux derivatives are treated explicitly at the old time level[26], but the new values are incorporated as soon as they become available. This explicit treatment of the spanwise flux terms enables the scheme to solve the three-dimensional equations by solving one spanwise station implicitly at a time. To eliminate any dependency the solution may have on the spanwise marching direction, the solver reverses the marching direction with every spanwise sweep.

The resulting left-hand-side of the matrix equation is approximately factored into alternating directions

$$
\begin{aligned}
&\left(\mathbf{I}+\Delta \tau \delta_{\xi} \mathbf{A}^{n}\right)\left(\mathbf{I}+\Delta \tau \delta_{\zeta} \mathbf{C}^{n}\right) \Delta \mathbf{Q}^{n+1} \\
&=-\Delta \tau\left[\begin{array}{l}
\left(\mathbf{E}^{n}-\mathbf{E} \mathbf{v}^{n, n+1}\right)_{\xi}+(\mathbf{F}-\mathbf{F v})_{\eta}^{n, n+1} \\
+\left(\mathbf{G}^{n}-\mathbf{G} \mathbf{v}^{n, n+1}\right)_{\zeta}
\end{array}\right]
\end{aligned}
$$

The implicit operating matrices are then diagonalized using a similarity transformation [27].

\section{Spatial Discretization}

Standard second-order central differencing is used for the spatial derivatives. Spectral-radiusscaled fourth/second-difference artificial dissipation [22] is added for stability and to eliminate oscillations near shocks. The viscous terms are evaluated using half-point central differencing so that the computational stencil for the stress terms uses only three nodes in each of the three directions.

\section{Hybrid Coupling}

Since the structured code has multi-block capability, the coupling procedure treats the unstructured portion of the hybrid meshes as a separate block. The blocks are solved independently with respect to each other. Explicit boundary conditions are updated at the end of each time iteration. The two codes are loosely coupled, essentially running independently of each other. The only interaction between the structured solver and the unstructured solver is through boundary conditions. The advantage of this formulation is that almost any two given codes can be coupled together. In this paper, a central-differencing, finite-difference procedure and an upwind cell-centered finite volume procedure are coupled. The structured solver is formulated in an inertial frame of reference where as the unstructured solver is in a blade-fixed coordinate system. For non-moving-boundary cases, such as pressure-driven turbine stator flows, there is no difference between the two formulations. However, for moving-boundary cases, care must be taken when transferring data between the two solvers depending on how boundary conditions are prescribed. For example, consider a wing traveling at a constant velocity. Since the structured solver assumes the wing is moving and the unstructured solver assumes the domain is fixed in space, the total energy must be converted between the two frames of reference. At the end of each iteration, mass, momentum, and energy values are communicated between the structured and unstructured solvers.

Since the unstructured solver is a cell-centered finite-volume scheme and the structured solver is a vertex-based finite-difference scheme, the unstructured and the structured grids are overlap by one layer at the boundary in order to minimize interpolation and extrapolation errors at the boundary. By overlapping the grids instead of abutting them against each other, the boundary nodes 
for the structured solver are interpolated from the interior cell center values of the unstructured solver. Similarly, the cell boundary face values of the unstructured solver are interpolated from interior structured nodes. An illustration of the overlapped grid in $2 \mathrm{D}$ is shown in Fig. 1.

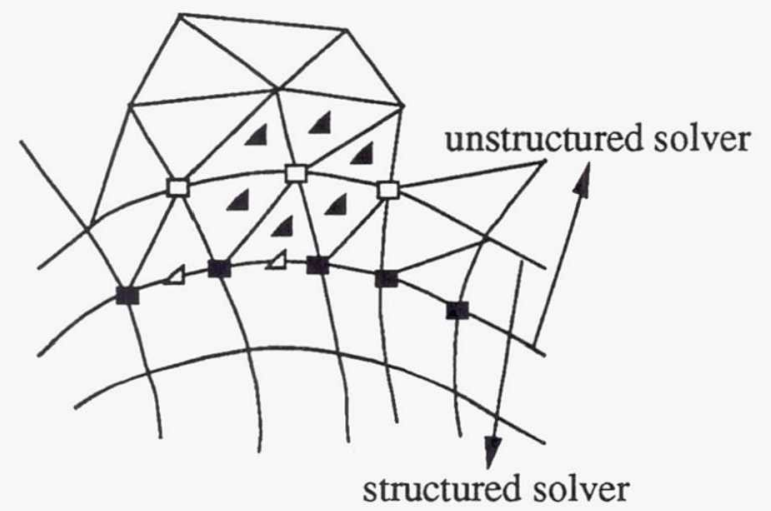

Figure 1. Structured-unstructured mesh overlapping by one layer.

Once the grid construction is completed, a table is generated to index unstructured nodes to their corresponding structured nodes, and vise-versa. The index is similar to the table generated between structured block boundaries for multi-block calculations.

\section{Results and Discussion}

Validation for both the structured [24] and the unstructured solvers $[28,17]$ have been documented previously and will not be presented here. To test the structured-unstructured hybrid analysis, calculations for a transonic inviscid flow over a fixed wing and a viscous flow for a turbine stator blade are presented.

For all calculations in this paper, a structured grid is first generated for the entire geometry. The unstructured grids are generated by subdividing a portion of the structured cells each into six unstructured tetrahedra (Fig. 2). This procedure simplifies grid generation and is suitable for the purpose of code validation.

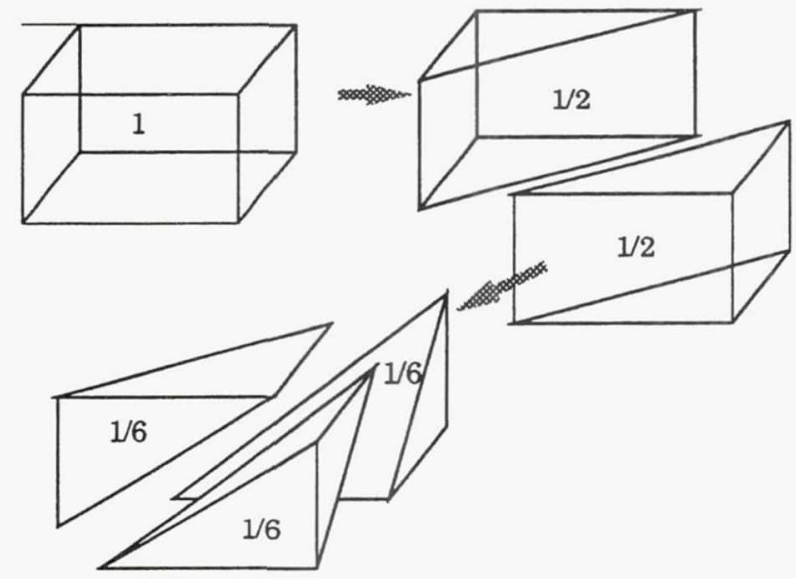

Figure 2. Unstructured tetrahedral cells generated using structured cells.

\section{Transonic Wing}

The first calculation selected for the hybrid scheme is a transonic wing at a freestream mach number of 0.8 and at $1.25^{\circ}$ angle-of-attack. For this test case, flow periodicity is imposed on the two endwall boundaries to simulate a two-dimensional flow.

A purely structured solution for this case is first obtained for comparison. An O-H grid topology is used; that is, an O-grid is generated at each spanwise station. For the structured calculation, each spanwise station consists of 121 (streamwise) x 41 (normal) points. Six spanwise stations are required due to the memory allocation algorithms for the multi-block structured solver. The number of spanwise stations used does not alter the results since the code simulates a two-dimensional problem.

For the structured-unstructured coupled procedure, the grid is divided into three zones. The inner zone, which wraps around the solid surface (121x12), is calculated using the structured solver. The middle zone (121x6), which wraps around the inner structured zone, is subdivided into tetrahedra for the unstructured solver. The outer zone, which extends from the middle zone to the free stream, is once again calculated using the structured solver. Fig. 3 illustrates the three-zones described for this case. 


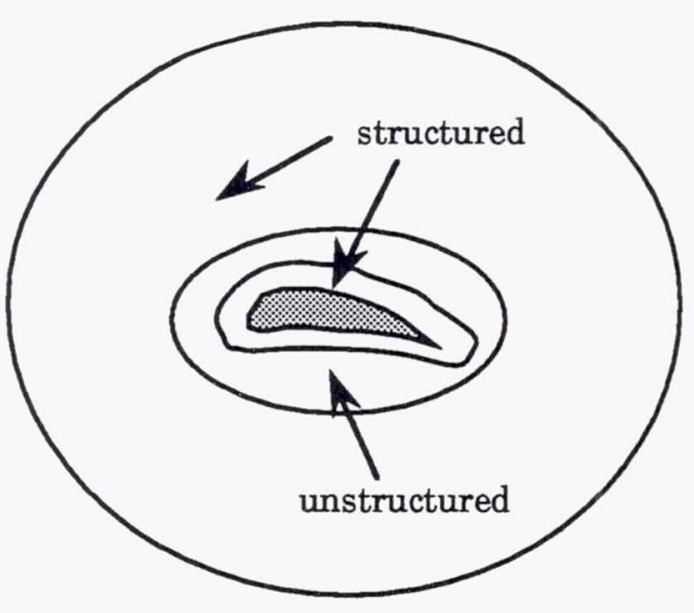

Figure 3. Structured-unstructured zones for the external wing calculation.

The actual grid used for the calculation is shown in Fig. 4.

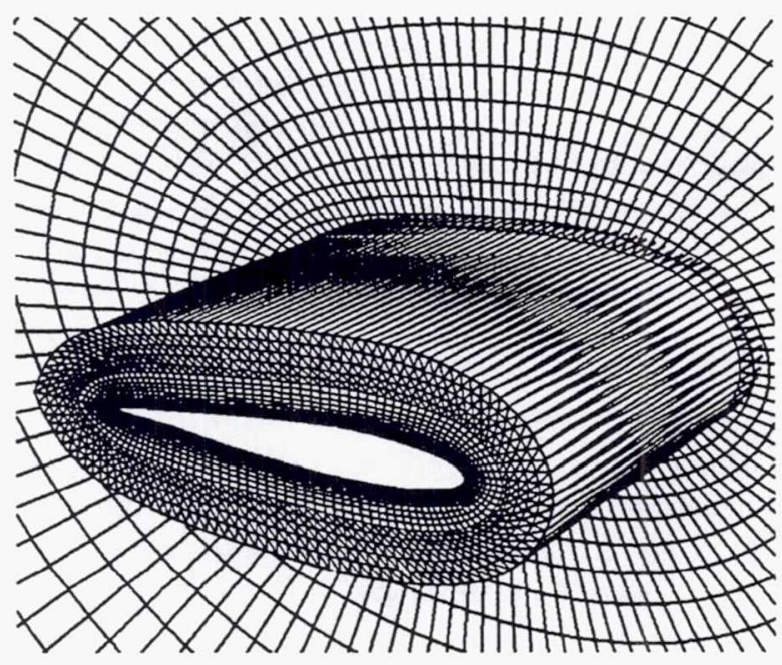

Figure 4. Structured-unstructured grid for the 2-D wing calculation.

The purpose of dividing the calculation into three zones is to simulate a Chimera type of grid where a simple background grid is generated for the entire domain and a structured grid is used around a solid object. Instead of interpolating between the two grids, an unstructured solver can be placed to connect the two set of grids and obtain node-to-node correspondence which remove the needs for interpolation. For a finite volume approach, this also insures flow conservation. This approach is also recommended for multi-body calculations. Many researchers have demonstrated structured- unstructured coupling for multi-element airfoil calculations where structured grids are place near the solid surfaces and an unstructured solver connects the structured grids and extends to the farfield. Since structured solvers are generally more efficient, it makes sense to minimize unstructured zones.

The unstructured solver can be run in both firstand second-order modes, while the structured solver is always second-order accurate. Surface pressure distributions for both first- and second-order hybrid solutions, as well as the fully structured solution are shown in Fig 5. Both hybrid solutions agree very closely with the standard structured solution, but the first-order unstructured solution exhibits a larger deviation on the lower surface.

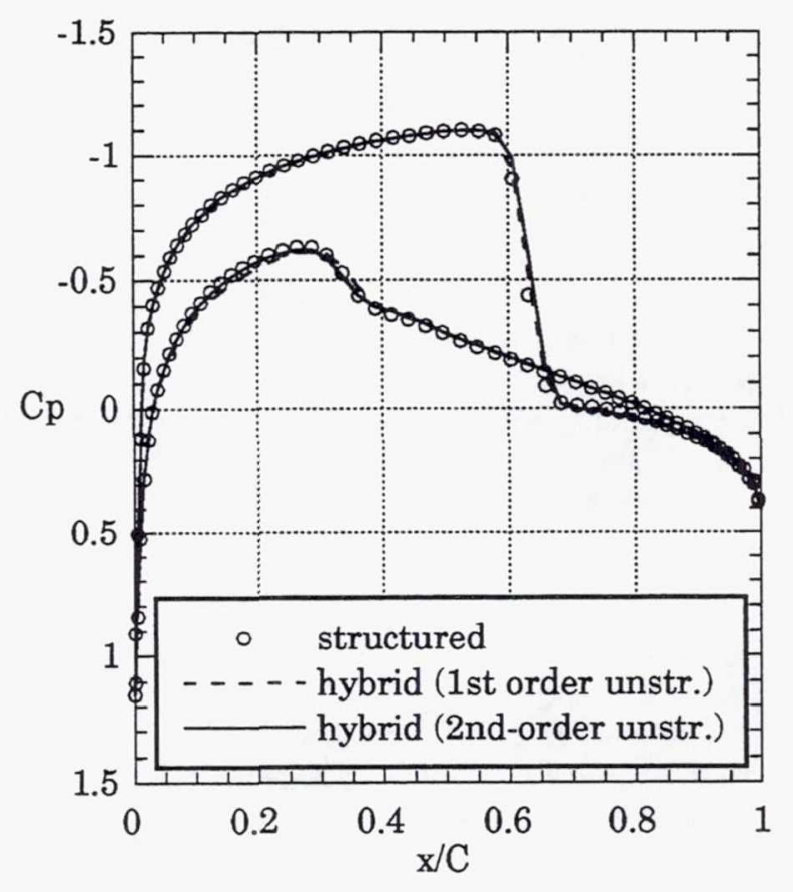

Figure 5. Surface pressure distribution.

To examine the coupling between the structuredunstructured solver across the three-zones, pressure contour lines are shown in Fig. 6 for the first-order and the second-order coupling, along with the single block structured solver for comparison. For the firstorder coupling, a slight discontinuity exists across the zonal boundary. However, the discontinuity is small and barely perceptible on a global scale. For the second-order coupling, the contours show the flow is smooth with little indication of the presence of the zonal boundaries. The mass flows in and out of the sandwiched unstructured zone are within $0.03 \%$ of each other, indicating that good flow conservation is 
achieved. The unstructured shock is somewhat more compact then the structured solver. One reason for this is that every structured cell becomes six tetrahedra so that in the streamwise direction, the unstructured cells are twice as dense as the structured cells. The present hybrid procedure converges considerably slower then the single structured grid due to the explicit formulation of the incorporated unstructured solver, which requires a low CFL number.

\section{3-D Viscous Flow Through Turbine Blades- Hybrid Procedure}

The second test case selected is a threedimensional annular cascade [29]. The geometry consists of an annular ring of 36 turbine stator vanes. The blades are $38.10 \mathrm{~mm}$ in span, untwisted, and of constant profile with an axial chord of $38.23 \mathrm{~mm}$. The stator has a tip diameter of $508 \mathrm{~mm}$ and a hub-to-tip radius ratio of 0.85 . The inlet flow angle is parallel to the axis of the cascade. The Reynolds number based on the inlet total quantities and axial chord length is 898,650 . The inlet total pressure and total temperature at one axial-chord length upstream of the blade leading edge are known from experiment. The exit hub static pressure to inlet total pressure ratio is 0.65 at 2.6 axial-chord lengths down stream of the blade trailing edge.

For the hybrid calculation, the computational domain extends one axial-chord length upstream of the blade leading edge and 2.6 axial-chord lengths down stream of the blade trailing edge. The exit hub static pressure to inlet total pressure ratio is known from experiment. The grid has 105 (inlet to exit) x29 (blade to blade) x15 (hub to tip) nodes and 23,000 cells are subdivided into unstructured cells. The structured grid wraps around the solid surfaces of the hub and the turbine blade while the rest of the domain is filled with unstructured cells. The hybrid grid at one radial station for the annular cascade is shown in Fig. 7. For the present grid, only 15 hub to shroud stations are used and the grid density near the trailing edge is very coarse (only 3 points defining the rounded trailing edge). While such a coarse grid is not acceptable for accurate prediction of viscous effects, it is sufficient for monitoring the communication between the structured and unstructured zones and to predict the overall flow field.

A no-slip boundary condition is applied at the hub and on the blade surfaces, and an inviscid slip condition is applied on the shroud. In the coupled calculation, the unstructured solver is run without turbulence modeling because the effects of turbulence are much reduced outside the boundary layer.

The pressure contour lines across the structured and unstructured zones are plotted in Fig. 8. The same degree of smoothness as in the previous wing case is observed. In Fig. 9, the chord wise surface static pressures normalized by the inlet total pressure are compared with experiment at $13.3 \%, 50 \%$, and $86.7 \%$ span. An overall agreement is found accept near the trailing edge where the computation on the present coarse grid did not pick up the peak values.

\section{Concluding Remarks}

A three-dimensional, unstructured, NavierStokes flow solver has been coupled with a threedimensional structured code to allow structuredunstructured hybrid calculations. The two codes are loosely coupled and interact only through boundary conditions. The structured solver is a centerdifferenced finite-difference scheme and the unstructured solver is an upwind-differenced finitevolume scheme.

The hybrid procedure has been tested for a transonic wing and an annular cascade, and good results have been obtained. The flow properties across the boundary between the structured and unstructured portions of the grid are smooth and well behaved. The results show that two distinct discretization techniques can be coupled together with little effect on the solution accuracy, as long as both are of same order.

\section{References}

1. Aronone, A., Liou, M-S., and Povinelli, A., "Transonic Cascade Flow Calculations Using Non-Periodic C-type Grids," CFD Symposium on Aeropropulsion, NASA Conference Publication 3078, 1990.

2. Barth, T.J., "A 3-D Upwind Euler Solver for Unstructured Meshes," AIAA Paper 91-1548, June 1991.

3. Batina, J.T., "A Fast Implicit Upwind Solution Algorithm for Three-Dimensional Unstructured Dynamic Meshes," AIAA Paper 92-0447, Jan. 1992. 
4. Frink, N.T., "Upwind Scheme for Solving the Euler Equations on Unstructured Tetrahedral Meshes," AIAA Journal, Vol. 30, No. 1, pp. 70-77, Jan. 1992.

5. Mavriplis, D.J., "Three-Dimensional Unstructured Multigrid for the Euler Equations," AIAA Journal, Vol. 30, No. 7, pp. 1753-1761, July 1992.

6. Dawes, W.N., "The Simulation of ThreeDimensional Viscous Flow in Turbomachinery Geometries Using a Solution-Adaptive Unstructured Mesh Methodology," ASME Paper 91-GT-124, 1991.

7. Spragle, G.S., Smith, W.A., and Yadlin, Y., "Application of an Unstructured Flow Solver to Planes, Trains, and Automobiles," AIAA Paper 93-0889, Jan. 1993.

8. Weatherill, N.P., "Mixed StructuredUnstructured Meshes for Aerodynamic Flow Simulation," Aeronautical Journal, pp. 111-123, Apr. 1990.

9. Mathur, S.R., Madavan, N.K., and Rajagopalan, R.G., "A Solution-Adaptive Hybrid-Grid Method for the Unsteady Analysis of Turbomachinery," ALAA Paper 93-3015, July 1993.

10. Soetrisno, M., Imlay, S.T., and Roberts, D.W., "A Zonal Implicit Procedure for Hybrid StructuredUnstructured Grids,” AIAA Paper 94-0645, Jan, 1994.

11. Nakahashi, K. and Obayashi, S., "FDM-FEM Zonal Approach for Viscous Flow Computation Over Multiple-Bodies," AIAA Paper 87-0604, Jan. 1987.

12. Kao, K.H. and Liou, M.S., "Direct Replacement of Arbitrary Grid-Overlapping by Non-Structured Grid," NASA TM-106601, May 1994.

13. Nakahashi, K., Nozaki, O., Kikuchi, K., and Tamura, A., "Navier-Stokes Computations of Two- and Three-Dimensional Cascade Flowfields," Journal of Propulsion and Power, Vol. 5, No. 3, pp.320-326, 1989.

14. Hwang, C.J. and Liu, J.L., "Inviscid and Viscous Solutions for Airfoil/Cascade Flows Using a Locally Implicit Algorithm on Adaptive Meshes," Journal of Turbomachinery, Vol. 113, pp.553-560, Oct. 1991.

15. Mathur, A., Sanjay, R., Madavan, B., Nateri, K., and Rajagopalan, R.G., "A Hybrid StructuredUnstructured Grid Method for Unsteady Turbomachinery Flow Computations," AIAA paper 93-0387, Jan. 1993.

16. Launder, B.E. and Spalding, D.B., "The Numerical Computation of Turbulent Flows," Computer Methods in Applied Mechanics and Engineering, Vol. 3, 1974, pp. 269-289.
17. Kwon, O.J. and Hah, C., "Solution of the 3-D Navier-Stokes Equations with a Two-Equation Turbulence Model on Unstructured Meshes Applied to Turbomachinery," AIAA Paper 941833, June 1994.

18. Roe, P.L., "Characteristic-Based Schemes for the Euler Equations," Annual Review of Fluid Mechanics, Vol. 18, pp. 337-365, 1986.

19. Holmes, D.G. and Connell, S.D., "Solution of the 2D Navier-Stokes Equations on Unstructured Adaptive Grids," AIAA Paper 89-1932, June 1989.

20. Frink, N.T., "Recent Progress Toward a ThreeDimensional Unstructured Navier-Stokes Flow Solver," AIAA Paper 94-0061, Jan. 1994.

21. Mavriplis, D.J., "Multigrid Solution of Compressible Turbulent Flow on Unstructured Meshes Using a Two-Equation Model," AIAA Paper 91-0237, Jan. 1991.

22. Jameson, A., Schmidt, W., and Turkel, E. "Numerical Solution of the Euler Equations by Finite-Volume Methods Using Runge-Kutta Time-Stepping Schemes," AIAA Paper 81-1259, June 1981.

23. Kunz, R.F. and Lakshminarayana, B., "Stability of Explicit Navier-Stokes Procedures Using k-e and k-e/Algebraic Reynolds Stress Turbulence Models," J. of Computational Physics, Vol. 103, pp. 141-159, 1992.

24. Tsung, F.-L. and Sankar, L.N., "Numerical Simulation of Flow Separation for Rotors and Fixed Wings,” AIAA paper 92-0635, Jan. 1992.

25. Baldwin, B.S., Lomax, H., "Thin-Layer Approximation and Algebraic Model for Separated Turbulent Flows," AIAA Paper 78-257, June 1978.

26. Rizk, Y.M. and Chausee, D.S., “ThreeDimensional Viscous-Flow Computations Using a Directionally Hybrid Implicit-Explicit Procedure," AlAA Paper 83-1910, 1983.

27. Pulliam, T.H. and Chaussee, D.S., "A Diagonal Form of an Implicit Approximate-Factorization Algorithm," J. of Computational Physics, Vol. 39, pp. 347-363, 1981.

28. Kwon, O.J. and Hah, C., "Three-Dimensional Unstructured Grid Euler Method Applied to Turbine Blades," AIAA Paper 93-0196, Jan. 1993.

29. Goldman, L.J. and McLallin, K.L., "Cold-Air Annular-Cascade Investigation of Aerodynamic Performance of Core-Engine-Cooled Turbine Vanes. I-Solid-Vane Performance and Facility Description," NASA TM X-3224, April 1975. 
first-order coupling

second-order coupling

single structured
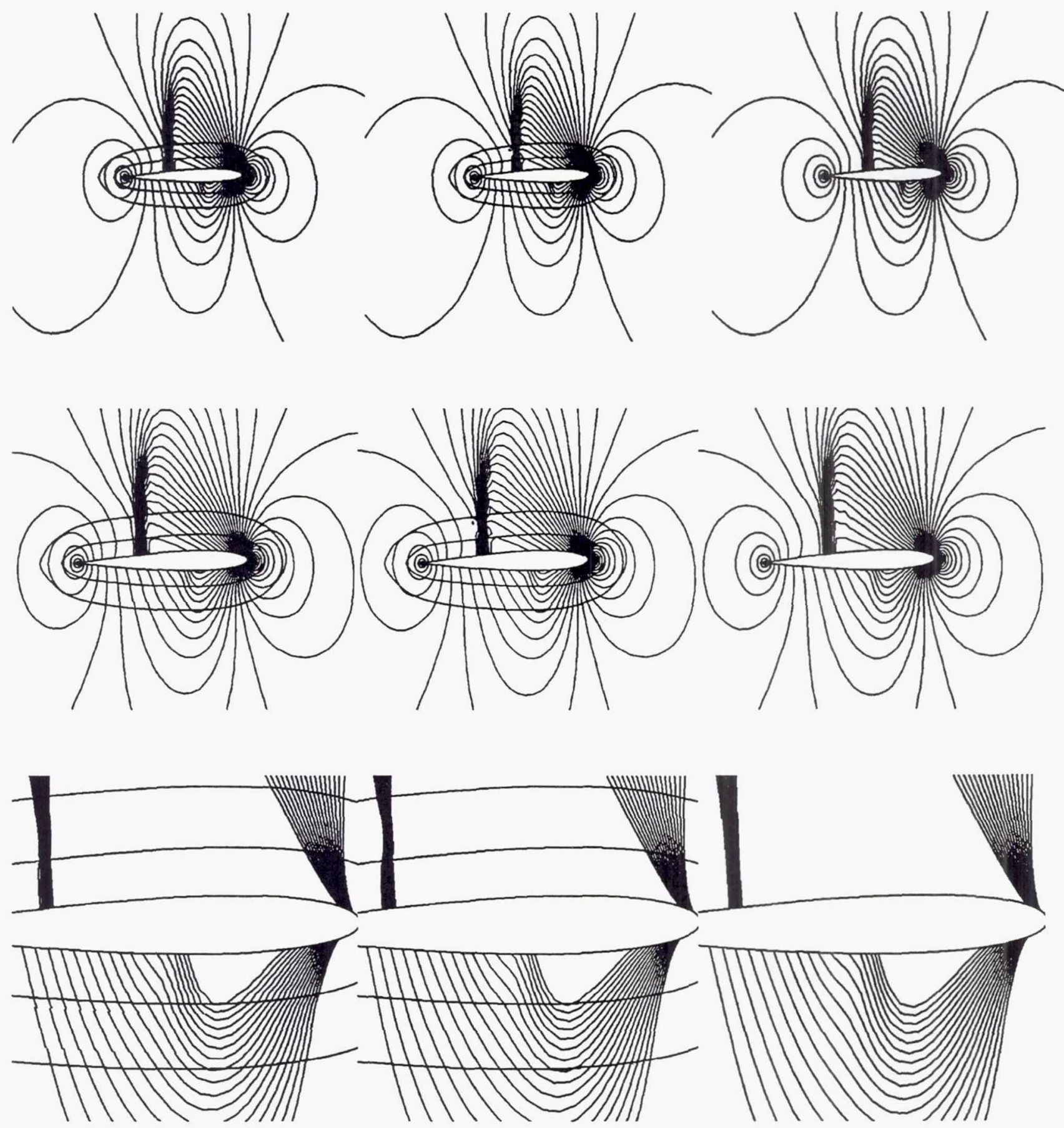

Figure 6. Pressure contour lines across the structured-unstructured zonal interface 


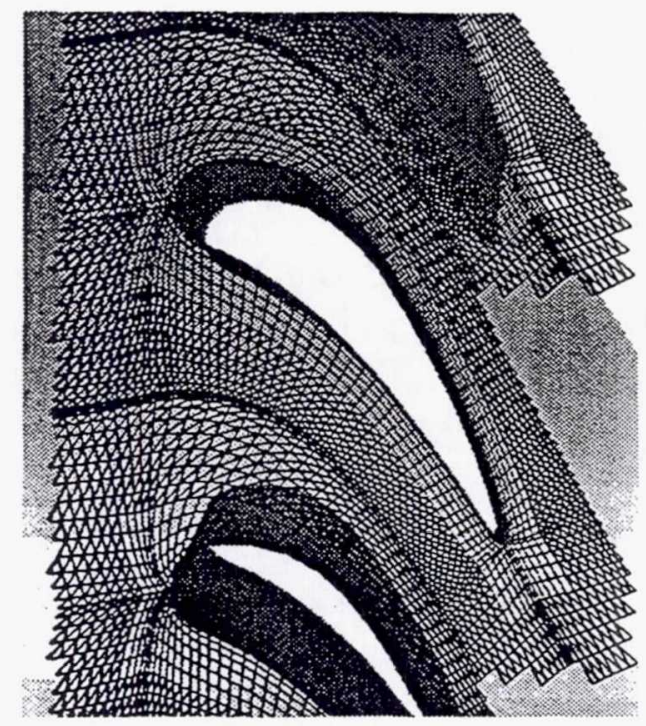

Figure 7. Structured-unstructured hybrid grid used for the annular cascade flow calculation.

(a)

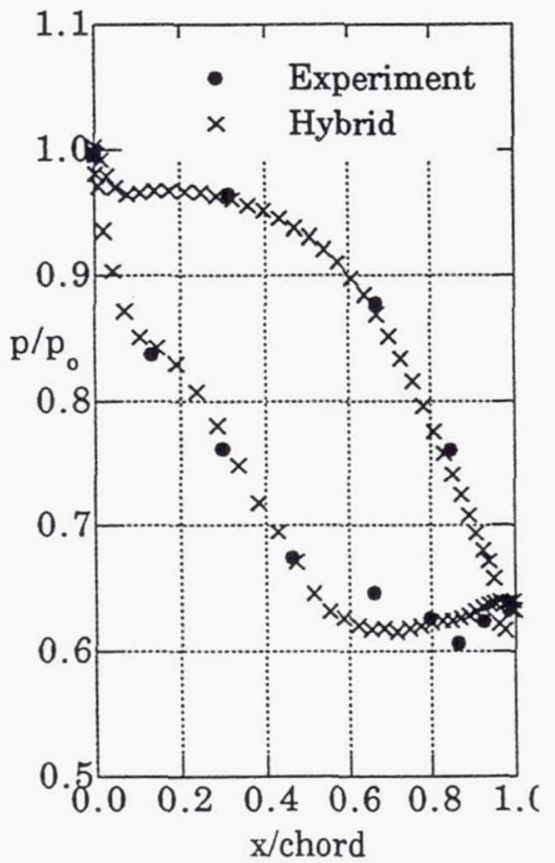

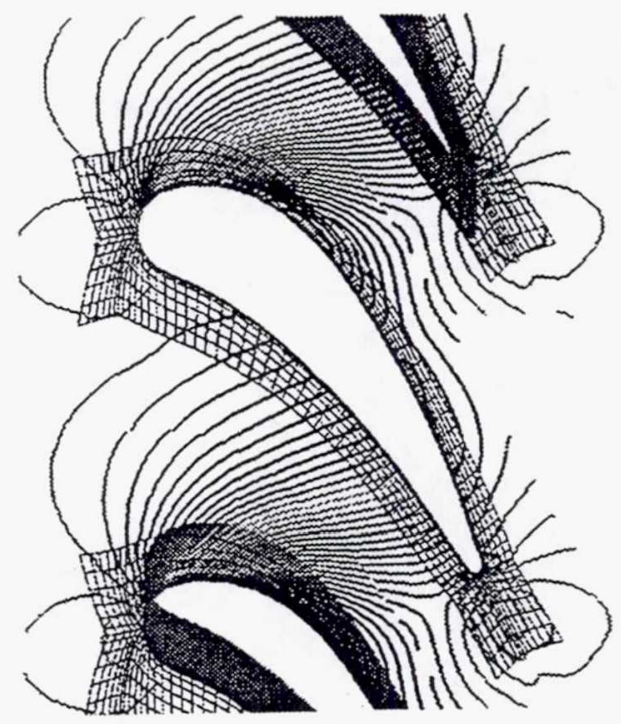

Figure 8. Pressure contour lines across the structured-unstructured zonal interface.

(b)

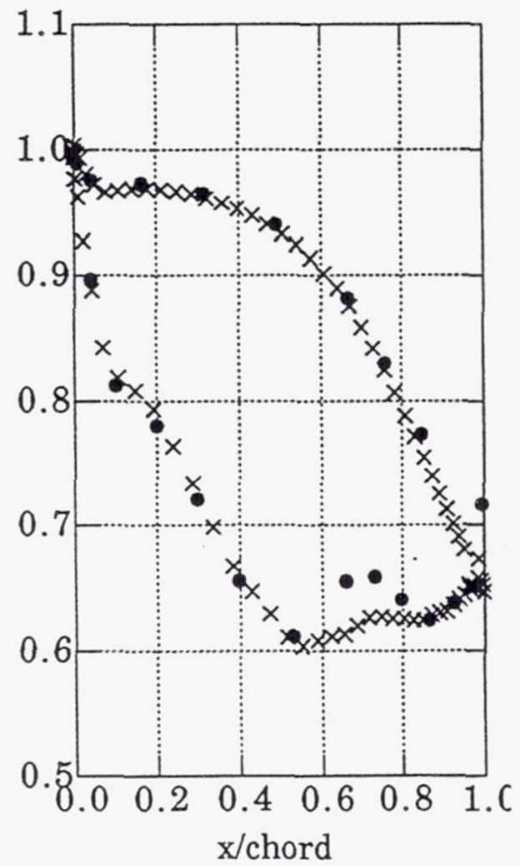

(c)

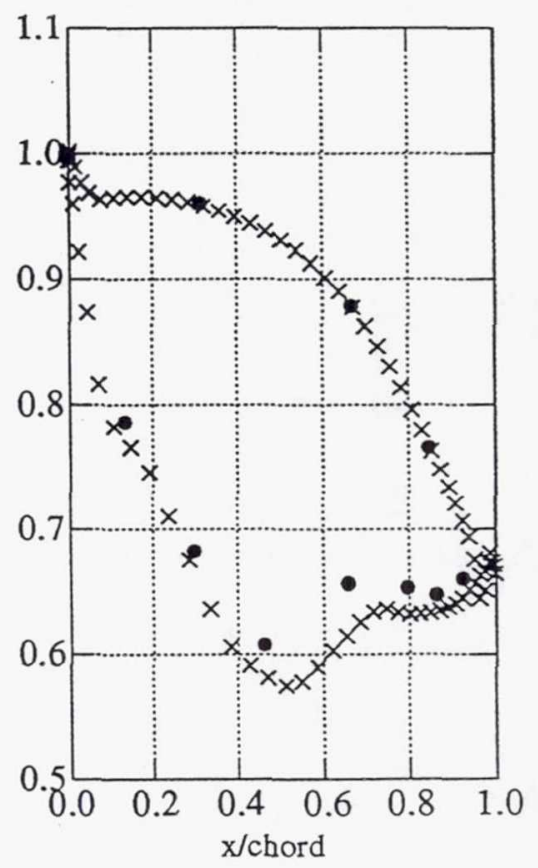

Figure 9. Static pressure distribution on the blade at $13.3 \%$ (a), $50.0 \%$ (b), and $86.7 \%$ (c) span. 
Public reporting burden for this collection of information is estimated to average 1 hour per response, including the time for reviewing instructions, searching existing data sources, gathering and maintaining the data Davis Highway, Suite 1204, Arlington, VA 22202-4302, and to the Office of Management and Budget, Paperwork Reduction Project (0704-0188), Washington. DC 20503.
1. AGENCY USE ONLY (Leave blank)
2. REPORTDATE
December 1994
3. REPORT TYPE AND DATES COVERED
Technical Memorandum

4. TITLE AND SUBTITLE

A Three-Dimensional Structured/Unstructured Hybrid Navier-Stokes Method for

Turbine Blade Rows

6. $A U T H O R(S)$

WU-505-90-5K

F.-L. Tsung, J. Loellbach, O. Kwon, and C. Hah

5. FUNDING NUMBERS

PERFORMING ORGANIZATION NAME(S) AND ADDRESS(ES)

National Aeronautics and Space Administration

Lewis Research Center

Cleveland, Ohio 44135-3191

8. PERFORMING ORGANIZATION REPORT NUMBER

E-9339

9. SPONSORING/MONITORING AGENCY NAME(S) AND ADDRESS(ES)

10. SPONSORING/MONITORING AGENCY REPORT NUMBER

National Aeronautics and Space Administration

Washington, D.C. 20546-0001

NASA TM-106813

ICOMP-94-31

AIAA-94-3369

\section{SUPPLEMENTARY NOTES}

Prepared for the 30th Joint Propulsion Conference and Exhibit cosponsored by AIAA, ASME, SAE, and ASEE, Indianapolis, Indiana, June 27-29, 1994. F.-L. Tsung and J. Loellbach, Institute for Computational Mechanics in Propulsion, NASA Lewis Research Center (work funded under NASA Cooperative Agreement NCC3-233); O. Kwon, NYMA, Inc., Engineering Services Division, 2001 Aerospace Parkway, Brook Park, Ohio 44142 (work funded by NASA Contract NAS3-27186); C. Hah, NASA Lewis Research Center. ICOMP Program Director, Louis A. Povinelli, organization code $2600,(216)$ 433-5818.

\begin{tabular}{l|l} 
12a. DISTRIBUTION/AVAILABILITY STATEMENT & 12b. DISTRIBUTION CODE
\end{tabular}

Unclassified - Unlimited

Subject Categories 34 and 64

This publication is available from the NASA Center for Aerospace Information, (301) 621-0390.

13. ABSTRACT (Maximum 200 words)

A three-dimensional viscous structured/unstructured hybrid scheme has been developed for numerical computation of high Reynolds number turbomachinery flows. The procedure allows an efficient structured solver to be employed in the densely clustered, high aspect-ratio grid around the viscous regions near solid surfaces, while employing an unstructured solver elsewhere in the flow domain to add flexibility in mesh generation. Test results for an inviscid flow over an external transonic wing and a Navier-Stokes flow for an internal annular cascade are presented.

\begin{tabular}{|l|l|}
\hline 14. SUBJECT TERMS \\
Navier-Stokes; Unstructured; Hybrid; Turbomachinery \\
\hline $\begin{array}{l}\text { 17. SECURTY CLASSIFICATION } \\
\text { OF REPORT } \\
\text { Unclassified }\end{array}$ & $\begin{array}{c}\text { 8. SECURTY CLASSIFICATION } \\
\text { OF THIS PAGE } \\
\text { Unclassified }\end{array}$ \\
\hline
\end{tabular}

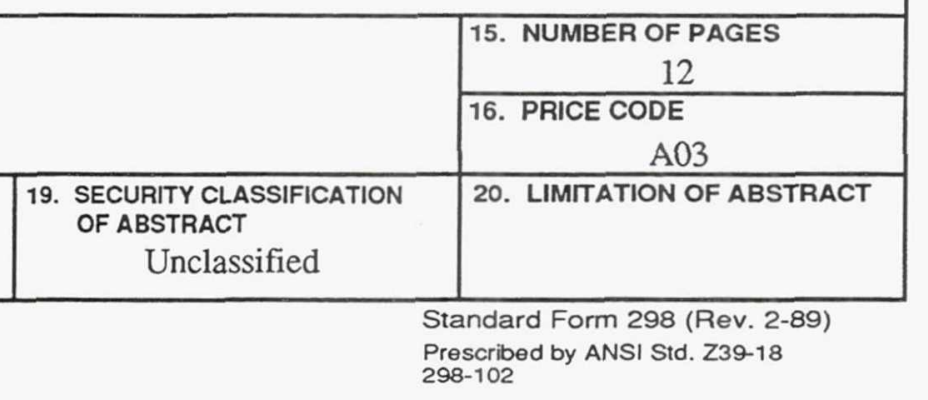

